Aim To look at the effect of the consultant delivered care in comparison to the registrar delivered care on the clinical services, patient care, satisfaction and staff opinion.

Method All the patients attending our paediatric assessment unit between 1130 am and 2100 were assessed on three consecutive days for two weeks. The parameters compared were the length of time taken to make a clinical decision from the time the patient was seen, investigations performed, any readmissions, patient outcome, any significant incidents, patient satisfaction and nursing staff opinion. Results A total of 51 patients were analysed. 21 of them were seen by the consultants and 29 were seen by the registrars. The time taken for Consultants to make the clinical decision and management plan varied from 15 to 100 minutes with a median of 30 minutes and mean of 39.6 minutes. The registrars took 90 to 480 minutes for the clinical decision with a median time of 250 minutes and mean of 233 minutes. 4 (19\%) and $16(55 \%)$ patients seen by consultants and registrars had investigations respectively. 2 patients, seen by registrars, with Paediatric Early Warning Score of more than 4 were admitted after $280 \mathrm{~min}$. One of these patients needed HDU admission and was transferred to specialist hospital. Another patient was diagnosed to have sepsis and had acute deterioration. 16 (76\%) patients were discharged home and 5 (24\%) patients were admitted by consultants. In the group seen by registrars or speciality trainees, 10 patients (34.5\%) were sent home, 17 were admitted (59\%) and 2 were transferred out. There was one readmission from the group seen by the speciality trainees Patients' satisfaction survey was similar in both groups. Nursing staff in POAU felt there was quicker decision making and more precise plans when seen by the Consultants. Conclusion The consultants made faster decision, performed less investigations and discharged more patients home with no readmissions or clinical incidents.

\section{G132(P) USE OF NASAL CPAP IN INFANTS WITH BRONCHIOLITIS IN THE SOUTH OF ENGLAND: A MULTICENTRE, PROSPECTIVE, OBSERVATIONAL STUDY}

doi:10.1136/archdischild-2013-304107.144

${ }^{1,2}$ A Saha, ${ }^{2} \mathrm{E}$ Vamvakiti, ${ }^{2} \mathrm{M}$ Linney. ${ }^{1}$ Department of Paediatrics, Maidstone and Tunbridge Wells Hospitals NHS Trust, Maidstone, UK.; ${ }^{2}$ Department of Paediatrics, Western Sussex Hospitals NHS Trust, Worthing, UK

Aim Bronchiolitis is a common respiratory illness in childhood with 64 million cases of RSV bronchiolitis worldwide every year. In England, 2.8\% hospital admissions in children $<1$ year of age are due to RSV bronchiolitis with upto $5 \%$ of these patients going on to develop respiratory failure.

The Aim of this study was to review the use of nasal continuous positive airway pressure (nCPAP) in infants with bronchiolitis. A secondary aim was to identify predictive factors for CPAP failure resulting in endotracheal intubation and mechanical ventilation

Methods A prospective, multicentre, observational study was undertaken from 1November 2008 to 28February 2009. Seven Paediatric Units in the South of England participated in the study. Data was collected on indications for nCPAP, respiratory rate and blood gases prior to nCPAP, total number of days on nCPAP and length of hospital stay. Results A total of 51 infants with the clinical diagnosis of bronchiolitis required nCPAP during the study period. The main indications were increased work of breathing (47.0\%), apnoeas (39.2\%) and increasing oxygen requirements (23.5\%). Among them 16 were ex-preterm $(31.37 \%)$. Prior to nCPAP the mean respiratory rate was $63 / \mathrm{min}$ (range 28 to 120 ), mean oxygen saturations $85 \%(70$ to $98 \%$ ), mean pH 7.25(7.03 to 7.36) and mean PCO2 10.42(4.36 to 19.0). The average time on nCPAP was 2.6 days( 2 hours to 11 days) and the average length of hospital stay was 10.96 days (5 to 22 days). There were no reported dealths. 11 out of the 51 infants failed trial on nCPAP requiring intubation $(21.5 \%)$. The main indications were apnoeas $(45.45 \%), \mathrm{CO}_{2}$ retention $(36.36 \%)$ and increasing work of breathing (36.36\%). Subgroup analysis revealed that 7 out of the 11 infants requiring intubation were ex-preterm (63.6\%) and 8 had significant PCO2 rise (>8) prior to the trial of nCPAP (72.72\%).

Conclusions nCPAP has a good success rate in bronchiolitis. In our study, predictive factors associated with nCPAP failure were prematurity and high $\mathrm{CO}_{2}$ retention prior to trial of nCPAP. The authors recommend the availability of nCPAP facilities in all paediatric units and appropriate training of medical and nursing staff in its optimal use.

\section{G133(P) MANAGEMENT OF PROLONGED JAUNDICE IN NURSE-LED CLINICS - 10 YEARS OF A SAFE AND EFFICIENT SERVICE}

doi:10.1136/archdischild-2013-304107.145

1. 2M Hyrapetian, 'R J Hewitson, 'H Mackinnon. 'General Paediatrics, The Whittington Hospital NHS Trust, London, UK; 2 Paediatrics, London School of Paediatrics, London, UK

Aims Prolonged jaundice in babies is common and usually harmless but a very small number of cases have serious pathology, such as biliary atresia, in which early detection is vital. Many "well" babies with prolonged jaundice undergo extensive investigations with a very low yield of important positive results. Our aim was to assess the safety, efficiency and cost effectiveness of a nurse-led prolonged jaundice clinic which has been in operation at our district general hospital for ten years, performing minimal investigations compared to standard practise.

Methods We collected data retrospectively for all babies $<3$ months of age in whom a conjugated bilirubin level was measured at $\geq 14$ days of age (term) or $\geq 21$ days (preterm) from January-August 2011, excluding babies on the neonatal unit. We reviewed clinic proformas completed for each baby seen with prolonged jaundice, medical notes and hospital results system to establish whether the baby was managed in the nurse led prolonged jaundice clinic or elsewhere by doctors, investigations undertaken and clinical outcomes.

Results 91\% (176) of 194 babies were managed in the nurse led prolonged jaundice clinic; 5.6\% (10) had additional investigations which identified one case with significant pathology. Of the 9\% (18) of babies seen elsewhere by doctors (A\&E/outpatients/inpatients/ daycare), 77\% (14) had additional investigations. 3 babies had serious pathology including one case of biliary atresia which presented late (48 days old).

Conclusion For the last ten years we have managed the vast majority of babies with prolonged jaundice in our nurse-led clinic, successfully avoiding unnecessary, time-consuming and expensive investigations for well babies whilst promptly identifying cases with serious pathology.

Our district general hospital has recently merged with two local community health services to form an Integrated Care Organisation, providing a unique opportunity to move the nurse-led prolonged jaundice clinic into the community. Based on our well established model, such clinics could be conducted in local health centres by midwives or community paediatric nurses, supervised by a consultant paediatrician. This would allow further cost savings and be more convenient for families.

\section{G134(P) LUMBAR PUNCTURES IN CHILDREN: A SURVEY OF CURRENT PRACTISE}

doi:10.1136/archdischild-2013-304107.146

${ }^{1,2}$ D Son, ${ }^{2}$ R Roots. ${ }^{1}$ Paediatrics, Epsom and St Helier University Hospitals NHS Trust, Carshalton, Surrey, UK: ${ }^{2}$ Medical School, St George's University of London, London, UK

Aims Lumbar puncture (LP) is a common procedure in children used to diagnose infection and various neurologic processes. We want to survey current clinical practises, in terms of positioning and use of analgesia, in paediatric LPs.

Methods A survey questionnaire was designed for distribution to staff on the wards of 10 paediatric departments in one city. The 